\title{
Ozone therapy in the management of cancer-treatment toxicity [abstract]
}

\author{
Bernardino Clavo1,2,3,a,b, Norberto Santana-Rodríguez¹, Pedro Llontop¹, Charlin \\ Méndez ${ }^{1}$, Keila Zerecero', Dolores Fiuza1
}

${ }^{1}$ Research Unit, 2Dep. Radiation Oncology and ${ }^{3}$ Chronic Pain Unit from the Dr. Negrin University Hospital. Las Palmas (Canary Islands), Spain. alCIC (Canary Islands Institute for Cancer Research), bGICOR (Research Group of Radiation Oncology)

\section{ABSTRACT}

\section{OPEN ACCESS}

\section{Citation}

Clavo B, Santana-Rodriguez N, Llontop P, Mendez C, Zerecero K, Fiuza D. Ozone therapy in the management of cancer-treatment toxicity [abstract]. Proceedings of The World Conference on Ozone Therapy in Medicine, Dentistry and Veterinary. Ancona (Italy). September 22nd - 23rd - 24th , 2017. J Ozone Ther. 2019;3(4):10. doi: 10.7203/ jo3t.3.4.2019.15399

\section{Academic Editor \\ Jose Baeza-Noci, \\ School of Medicine, Valencia University, SPAIN}

\section{Editor}

World Federation of Ozone Therapy, Bolgna, ITALY

\section{Received}

June 17, 2019

\section{Accepted}

December 08, 2019

Published

December 30, 2019

\section{Intellectual Property}

Bernardino Clavo.

This is an open access article distributed under the terms of the Creative Commons Attribution License (CC BY 4.0), which permits unrestricted use, distribution, and reproduction in any medium, provided the original author and source are credited.

\section{Author Information}

Email: bernardinoclavo@gmail.com
Purpose: This presentation will be focused to show clinical and preclinical works using Ozone therapy in the management of side effects related to cancer treatments

Material and methods: Published articles and our experience about the potential beneficial effects of Ozone therapy in different adverse events related with cancer treatments.

Results: Clinical experiences have shown that Ozone therapy can decrease or avoid delay in the commencement of Radiotherapy and Chemotherapy in patients with delayed wound-healing after cancer surgery. These delays could lead to increased risk of tumor relapse.

Clinical studies have shown the success of Ozone therapy to treat different side effects of Radiotherapy, Chemotherapy and Surgery. In our institution Ozone therapy was used to treat: delayed healing after chemotherapy extravasation, radiation-induced dermatitis, fistula and fibrosis after cancer treatments, radiation- induced side effects such as: delayed-healing, vaginitis, cystitis, proctitis, brain injury. Other institutions have also described beneficial effects of Ozone therapy in the treatment of mucositis, wound infections or bisphosphonate-related jaw osteonecrosis in cancer patients

Finally, several preclinical studies have shown "protective" effect of Ozone preconditioning against selective toxicity induced by different chemotherapy drugs and radiation. These works suggest potential clinical application to prevent toxicity.

Discussion and Conclusions: Several studies support the potential role of Ozone therapy as complementary treatment to avoid delay in the commencement of Radio-Chemotherapy and prevent or treat some side effects of cancer-treatments. However, as it happens with hyperbaric chambers and other more accepted therapies, well addressed clinical trials are required for a definitive demonstration. Meanwhile, our work with Ozone therapy looking for the improvement of quality of life and/or symptom management has to follow an appropriate information to cancer-patients. 